\title{
Anterior cingulate activity during error and autonomic response
}

\author{
Hugo D. Critchley, ${ }^{\mathrm{a}, \mathrm{b}, \mathrm{c}, *}$ Joey Tang, ${ }^{\mathrm{b}}$ Daniel Glaser, ${ }^{\mathrm{b}}$ Brian Butterworth, ${ }^{\mathrm{b}}$ and Raymond J. Dolan ${ }^{\mathrm{a}}$ \\ ${ }^{\mathrm{a}}$ Wellcome Department of Imaging Neuroscience, Institute of Neurology, UCL, 12 Queen Square, London WC1N 3BG, UK \\ ${ }^{\mathrm{b}}$ Institute of Cognitive Neuroscience, UCL, 17 Queen Square, London WC1N 3AR, UK \\ ${ }^{\mathrm{c}}$ Autonomic Unit, National Hospital for Neurology and Neurosurgery and Institute of Neurology, Queen Square, London WC1N 3BG, UK
}

Received 17 December 2004; revised 29 April 2005; accepted 5 May 2005

Available online 5 July 2005

\begin{abstract}
The contribution of anterior cingulate cortex (ACC) to human cognition remains unclear. The rostral (rACC) and dorsal (dACC) ACC cortex are implicated in tasks that require increased response control due to emotional and cognitive interference, respectively. However, both RACC and AACC are activated by conditions that induce changes in visceral arousal, suggesting that ACC supports a generation of integrated bodily responses. To clarify the relationship between purely cognitive and psychophysiological accounts of ACC function, we scanned 15 subjects using functional magnetic resonance imaging while they performed numerical versions of the Stroop task. To index autonomic arousal, we simultaneously measured pupil diameter. Performance errors accounted for most of the variance in a pupil-derived measure of evoked autonomic arousal. In analysis of the functional imaging data, activity within a region spanning $\mathrm{rACC}$ and dACC predicted trial-by-trial variation in autonomic response magnitude and was enhanced during error trials, shown using conjunction analyses. Activity within other loci within rACC predicted evoked autonomic arousal and showed sensitivity to errors but did not meet criteria for both. These data highlight the role of ACC in psychophysiological aspects of error processing and suggest that an interface exists within ACC between cognitive and biobehavioral systems in the service of response adaptation.
\end{abstract}

(c) 2005 Elsevier Inc. All rights reserved.

Keywords: Anterior cingulate cortex; Error processing; Autonomic response; Pupil; Stroop

\section{Introduction}

Anterior cingulate cortex (ACC) is a large medial cerebral structure surrounding the anterior third of the corpus callosum. Despite remarkable homology in macroscopic and cellular ACC structure across species, its functional role is poorly understood.

\footnotetext{
* Corresponding author. Wellcome Department of Imaging Neuroscience, Institute of Neurology, UCL, 12 Queen Square, London WC1N 3BG, UK. Fax: +44 2079168517 .

E-mail address: h.critchley@fil.ion.ucl.ac.uk (H.D. Critchley).

Available online on ScienceDirect (www.sciencedirect.com).
}

Influential models associate regions of human ACC with attentional and cognitive processes, in addition to emotional and visceral functions ascribed to ACC in other species.

Attempts have therefore been made on the basis of neuroimaging evidence to parse ACC into distinct (cognitive, emotional and visceral) functional components. However, anatomically, ACC presents a distinct and chiefly homogeneous structure rather than a set of cytoarchitectonically defined 'modules'. Brodmann (1909) recognized only three subregions: subgenual area 25 and two areas that together stretch around the callosal genu to a transition zone with posterior cingulate at level of the central sulcus, labeled area 24 (area cingularis anterior ventralis, on the inside of the cingulate sulcus) and area 32 (area cingularis anterior dorsalis, on the outer rim of the cingulate sulcus). Recent histological studies extend this parcellation of human ACC to subgenual components of area 24 and 32 and a 'vertical' stratification of area 24 (Vogt et al., 1995; Öngür et al., 2003). Furthermore, a caudal ACC transition zone is now viewed as a distinct subregion (Vogt et al., 2003).

From a connectional perspective, ACC subregions are intrinsically interconnected (Morecraft and Van Hoesen, 1998). There are broad differences in the relative connectivity between inner area 24 (with other limbic structures and basal ganglia) compared to outer area 32 (with motor regions), however, areas 24 and 32 have close reciprocal connections (Barbas et al., 2003; Öngür and Price, 2000). Subgenual, genual and supragenual subregions of rostral ACC (rACC) interconnect closely with each other (Öngör and Price, 2000), with adjacent medial orbitofrontal cortex and with nearby regions implicated in autonomic control, emotional and appetitive behaviors, including hypothalamus, brainstem and nucleus accumbens (Öngör and Price, 2000; Barbas et al., 2003). The supragenual ACC merges caudally into a dorsal ACC (dACC) region that also interconnects with neighboring lateral prefrontal and premotor cortices and supplementary motor area (SMA) (Paus et al., 2001; Luppino et al., 2003; Takada et al., 2004). Caudally, ACC 'motor' cingulate areas interconnect with motor cortices, lateral basal ganglia and spinal motor units (Tachibana et al., 2004). However, these broad differences in external connectivity of rACC, dACC and caudal ACC regions do not convincingly indicate functional heterogeneity. In primates, afferents from all 
ACC regions probably converge on motor cingulate cortex (Morecraft and Van Hoesen, 1998), although evidence for a direct projection from cingulate gyrus (area 24) to motor cingulate areas is equivocal (Dum and Strick, 1993). However, rostral, dorsal and even motor ACC regions project to brainstem autonomic centers particularly (Vilensky and van Hoesen, 1981; An et al., 1998), and both $\mathrm{rACC}$ and $\mathrm{dACC}$ project to hippocampus via a posterior cingulate pathway (Morris et al., 1999).

While neuroanatomical studies suggest homogeneity within areas 24 and 32, neuroimaging studies suggest rostrocaudal functional parcellation of ACC. Supragenual rACC and dACC regions show enhanced activity in many effortful or attentiondemanding tasks (Paus et al., 1998; Duncan and Owen, 2000), whereas subgenual ACC regions generally show decreased activity (Raichle et al., 2001; Gusnard et al., 2001; Simpson et al., 2001). Such activity decreases are negatively correlated with sympathetic autonomic arousal (Matthews et al., 2004; Nagai et al., 2004a). A further distinction is also proposed between "cognitive" dACC and "emotional" rACC (including all regions surrounding the genu) (Bush et al., 2000). This distinction seems critically dependent on task and response. For example, emotional experiences engendered by anticipatory anxiety or pain activate the "cognitive" dACC region rather than the predicted genual "emotional" rostral ACC (Büchel et al., 1998; Rainville, 2002; Ploghaus et al., 1999, 2003).

Enhanced activity in dACC is observed using fMRI during many cognitive challenges. One explanation is that dACC activity is important to attention. Electroencephalographic (EEG) evidence implicates dACC as a generator of 'attentional' event-elated potentials (ERPs) during orientation, anticipation and response suppression (Nagai et al., 2004b). Neuroimaging studies also implicate dACC in attention-demanding executive control processes, particularly where stimuli specify a response choice (Posner and Petersen, 1999; Frith, 2001). A powerful line of evidence suggests that dACC is specifically engaged in processing conflict between competing stimuli or responses, as typified in tasks such as the Stroop color-naming task (Carter et al., 1995, 1998). Detection of response conflict is important in monitoring performance (Botvinick et al., 2001), and it is noteworthy that neuroimaging and ERP data show dACC sensitivity to errors in performance (Kiehl et al., 2000; Menon et al., 2001; Dehaene et al., 1994). This error-related activity reflects the rate and predictability of both external and internal error signals (Paulus et al., 2002; Holroyd et al., 2004). The functional significance of error-related negative potentials (ERN) to error detection is called into question by the observation that ACC damage may attenuate ERNs while error detection remains intact (Stemmer et al., 2004). Conflict detection and error monitoring are related processes, with errors occurring more frequently on trials with high response conflict. However, the observation that left dACC damage attenuates error-related ERPs (and causes deficits in error correction) yet leaves conflict-related ERPs intact (Swick and Turken, 2002) suggests further neuroanatomical dissociation.

Many neuroimaging and electrophysiological studies report correlates within ACC of a number of cognitive processes. However, lesion data provide only equivocal data to suggest a primary function of ACC in attention, executive function or cognitive control (Turken and Swick, 1999; Stuss et al., 2001; Rushworth et al., 2004; Fellows and Farah, 2005). Executive or cognitive control functions may be entirely normal in the context of large lesions involving ACC (Critchley et al., 2003; Fellows and
Farah, 2005). ACC is a major component of MacLean's visceral limbic system, connected throughout its extent with autonomic nuclei (Vilensky and van Hoesen, 1981; Morecraft and Van Hoesen, 1998; An et al., 1998; Öngör and Price, 2000; Barbas et al., 2003). Moreover, activity in rostral and dACC regions correlates with (and predicts) cardiovascular and electrodermal arousal evoked by a range of cognitive, emotional and motivational tasks (Fredrikson et al., 1998; Critchley et al., 2000, 2001a,b, 2003; Gianaros et al., 2004; 2005). Damage to rostral and dorsal ACC impairs efferent sympathetic drive (Zahn et al., 1999; Critchley et al., 2003), whereas activity in subgenual cingulate shows an inverse relationship with tonic measures of sympathetic arousal (Matthews et al., 2004; Nagai et al., 2004a).

The above observations, we suggest, provide for an alternative hypothesis of an autonomic contribution to ACC function. Specifically, we have proposed that ACC integrates somatosensory, interoceptive, cognitive and motivational states with states of bodily arousal (Critchley et al., 2003), an integrative process that may underpin autonomic psychophysiology. Thus, errors can trigger autonomic arousal responses associated with electrophysiological potential putatively originating in ACC (Hajcak et al., 2003). We undertook a functional neuroimaging experiment to explore further the function of anterior cingulate cortex and how this may be reflected in regional brain activity measured using fMRI. We predicted that during Stroop task performance ACC activity would reflect variance associated with task-induced changes in autonomic arousal rather than stimulus properties such as response conflict that might engender activity in regions such as the supplementary motor area (SMA). We therefore used functional magnetic resonance imaging (fMRI) to test the hypothesis that control of autonomic states of arousal provides a robust unitary account of ACC activity. We used a measure of pupillary response to provide a rapid index of changes in autonomic activity induced during cognitive processing. Correlates of central cognitive influences on pupillary response have been hitherto underexplored.

\section{Materials and methods}

\section{Subjects}

Fifteen healthy right-handed subjects were recruited to take part in a study at the Wellcome Department of Imaging Neuroscience. These volunteers gave fully informed consent which was approved by the local ethics committee. Each participant was screened to exclude medication and conditions including psychological or physical illness or history of head injury. Mean age $( \pm \mathrm{SD})$ of participants was $23 \pm 3$ years.

\section{Experimental design and task}

We used functional magnetic resonance brain imaging (fMRI) to examine regional brain activity during performance of modified (numerical) Stroop tasks. Our index of autonomic arousal was derived from stimulus-induced influences on the magnitude and recovery of the pupil light reflex. The light reflex is reflex contraction of the sphincter muscle of the iris in response to light causing the pupil to become smaller. The magnitude and form of parasympathetic pupil response are influenced by both tonic and phasic changes in sympathetic and parasympathetic activity. 
Subjects were scanned in the dark, with intermittent visual projection of isoluminant stimuli and display of a fixation cross providing the only ambient illumination. Each participant underwent two fMRI scanning sessions while performing two versions of a numerical Stroop task. The sessions were counterbalanced across subjects. Each session consisted of 192 trials with a further 25 blank (null) trials. Trials were presented in counterbalanced pseudorandom order every $3 \mathrm{~s}$. In both the tasks, the subject viewed the stimuli, a pair of numbers, on a projection of a computer screen and made reaction-time responses via a button box held in both hands, responding to the left with a left index finger button press and responding to the right with a right index finger button press. In the Numerical task session, subjects made numerical judgments. Thus, on each trial, the subject saw a pair of numbers presented side by side and had to make a rapid button press response to the side of the numerically higher (while ignoring distraction from differences in the physical sizes) of the two numbers. Similarly, in the Physical task, subjects made physical judgments of the relative sizes of the stimuli, responding to the physically largest stimulus, while ignoring distraction from differences in the numerical values of the stimuli (Fig. 1). These tasks were developed behaviorally by rigorous pilot testing to provide corresponding degrees of difficulty and conflict in numerical and physical tasks (Tang et al., submitted for publication). Before scanning, the subject familiarized with the computerized task events and were trained to maintain visual fixation. Tasks were written and presented, and behavioral responses were logged via a desktop computer running Cogent software on a Matlab platform (Mathwork, Nantick MA). It was not the purpose of the present study to examine differences between Numerical and Physical Stroop tasks, reported elsewhere (Tang et al., submitted for publication), but to use the Stroop task performance to directly address the neural correlates of the relationship between conflict error and autonomic response.

\section{Physiological monitoring and post-processing}

Autonomic arousal responses are reflected across different bodily organs, which generally (but not necessarily) correlate. Pupillometry was used to index event-related autonomic arousal during Stroop task performance on the basis of novelty (correla-

Physical judgment

$\begin{array}{rrrrrr}\text { incongruent } & 6 & 8 & \text { incongruent } & 6 & 8 \\ \text { congruent } & 4 & 9 & \text { congruent } & 4 & 9 \\ \text { neutral } & 4 & 4 & \text { neutral } & 9 & 4\end{array}$

Fig. 1. Task stimuli. Subjects performed two separate Stroop tasks in which two-choice reaction time decisions were made to number stimuli on the basis of numerical value (Numerical Stroop) or physical size of the symbol (Physical Stroop). The format of the stimuli was identical for both tasks. Subjects maintained central fixation, and numbers were placed to the left and right of the fixation cross. Subjects responded with left or right key press. In the Numerical task, discrepancies in the size of stimuli provided congruent, neutral or incongruent interference; similarly for the Physical Stroop task, differences in physical magnitude provided congruent, neutral or incongruent interference. The figure illustrates equivalent visual stimuli in congruent, incongruent and neutral trials of numerical and physical tasks. tions of brain responses with electrodermal and cardiovascular arousal have been previously described) and convenience since pupil responses provide rapid discrete arousal events that are less prone to habitation and baseline drift compared to electrodermal responses (Critchley et al., 2002; Nagai et al., 2004a). Moreover, previous studies have reported association between ACC activity and both cardiovascular (Critchley et al., 2000; 2001a, 2003; Gianaros et al., 2004, 2005) and sympathetic skin responses (Fredrikson et al., 1998; Critchley et al., 2001b; Nagai et al., 2004a), yet it is uncertain whether pupil autonomic arousal mechanisms show corresponding functional relationship. Eye movements and pupil diameter were continuously monitored during fMRI scanning using infrared eye tracking (Applied Sciences Laboratories, Waltham MA, Model 504). Light flux in the scanning environment was constrained to video presentation of isoluminant stimuli interleaved with presentation of a fixation cross. Pupil data were recorded simultaneously with the task events at $80 \mathrm{~Hz}$. Pupil data were processed post hoc in Matlab to remove blink responses and periods of non-fixation or poor signal. The pupil time series was interpolated at $1000 \mathrm{~Hz}$ to facilitate accurate synchronization with stimulus onset timing, temporally smoothed (full-width half maximum $20 \mathrm{~ms}$ ) and assigned to each individual trial or null trial. To provide a standard measure of autonomic response, individual trial responses were zeroed at the $0.5 \mathrm{~s}$ baseline period prior to stimulus presentation. The $0.5 \mathrm{~s}$ recovery period following light reflex, beginning at the offset of the stimulus (i.e. $1 \mathrm{~s}$ into the trial), was quantified for each trial. This measure (post-stimulus redilatation following the light reflex) of trial-bytrial changes in pupil size was chosen to represent evoked autonomic arousal, reflecting both a parasympathetic blunting of the light reflex and the onset of sympathetic influences on the recovery from the light reflex (Smith, 1992) while correcting for temporal drift in autonomic tone (Fig. 2A). Sympathetic arousal influences on pupil size are dilatory and manifest within the steeper gradient of recovery and (often) rebound (Fig. 2B). The relative attenuation of the light reflex at each trial (no null events) was used as a parametric modulator of that trial. Each trial was thus modeled as a delta function where height reflected the pupil response. This regressor was transformed into a session-specific continuous covariate of interest by convolving with a canonical hemodynamic response function and interpolated at scan frequency before being entered into the design matrix. This approach allowed us to examine event-related neural activity associated with stimulusevoked autonomic changes (measured at the onset of pupil redilatation) independent of trial categorization into congruent, incongruent, neutral and error. Moreover, trial-by-trial association of activity changes with evoked changes in pupil response provides a degree of control for tonic influences of autonomic arousal state including that induced by performance anxiety (habituating over the task session).

\section{Functional imaging data acquisition, pre-processing and analysis}

Participants were scanned at $1.5 \mathrm{~T}$ (Siemens Sonata, Erlangen Germany) performing the experimental task over two counterbalanced sessions. T2*-weighted echoplanar images optimized for blood-oxygenation-level-dependent (BOLD) contrast were acquired (TE $50 \mathrm{~ms}$, TRvol $2.52 \mathrm{~s}, 28 \times 3.5 \mathrm{~mm}$ thick slices, tilt $-30^{\circ}$ ). A preparation pulse (duration $1 \mathrm{~ms}$, amplitude of $-2 \mathrm{mT} /$ $\mathrm{m})$ was used in the slice selection direction to compensate for through-plane susceptibility gradients enhance imaging of regions 

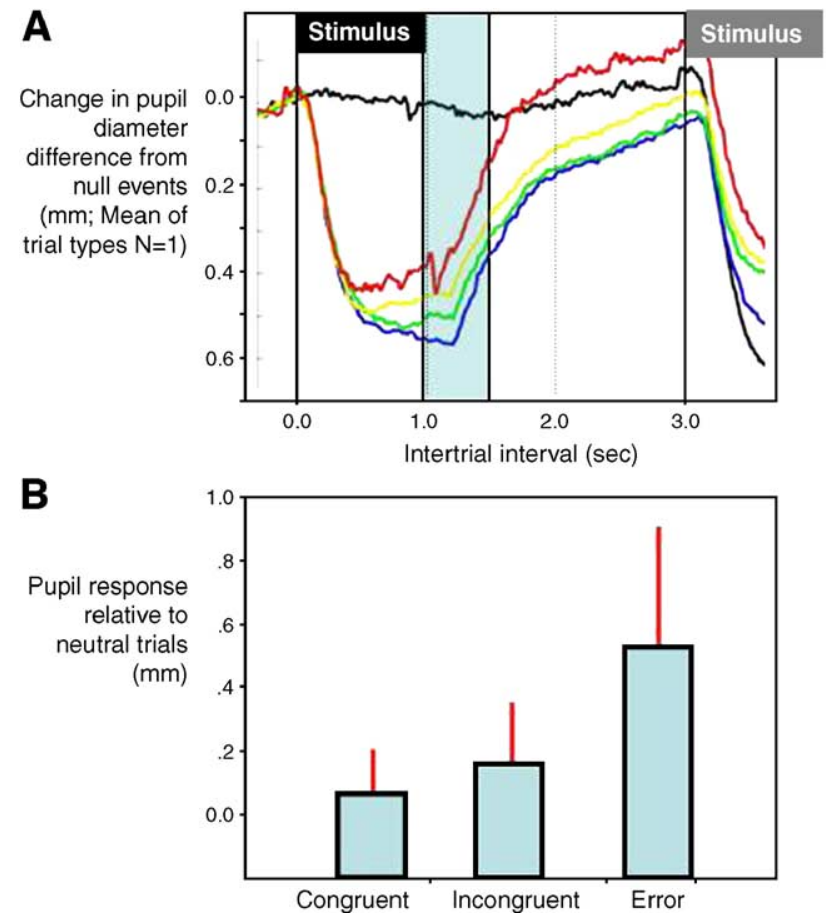

Fig. 2. Pupil measurements of arousal. Pupil constriction occurred to presentation of each stimulus, representing the pupil light reflex. Typically, when pupil responses are influenced by non-luminance properties of the stimuli (e.g. on viewing emotionally arousing stimuli), this results in attenuation of the light reflex and relative pupil dilation. These processes reflect sympathetic autonomic influences on the pupil. In our experiment, we continuously recorded pupil size throughout Stroop task performance and derived trial by trial time courses of changes in pupil diameter from the eyeblink-removed pupil dataset. Trial-specific measures of pupil size represented baseline-corrected mean responses in the $500 \mathrm{~ms}$ period following stimulus offset. Panel (A) illustrates the mean pupil changes for each trial type in one subject. No change is observed for blank trials (null events; black line). For remaining trials, $1 \mathrm{~s}$ stimulus presentation results in pupil constriction and subsequent recovery. Pupil constriction is most marked for neutral trials (blue line), congruent trials are given in green, incongruent correct trials in yellow and error trials in red. Error trials resulted in the greatest pupil arousal response measured over the $500 \mathrm{~ms}$ post-stimulus period (shaded). (B) Across all subjects, the mean ( $\pm \mathrm{SE}$ ) differences in the pupil response to congruent, incongruent and error trials, compared to neutral trials, are plotted. Data from 2 sessions (two different subjects) were excluded as they contained only 2 and 1 errors. On average, error trials enhanced pupil arousal response relative to other trial types.

including orbitofrontal and medial temporal lobe and periventricular regions traditionally prone to dropout or distortion (Deichmann et al., 2003).

Image pre-processing and subsequent analyses were undertaken using statistical parametric mapping (SPM2) (http://www.fil.ion. ucl.ac.uk/ spm/SPM2.html) on a Matlab platform. Images were initially realigned and unwarped, correcting for motion artefact. Differences in the timing of image slices across each individual volume were corrected, and each volume was transformed into standard stereotaxic space and smoothed with a Gaussian filter (full-width half maximum $8 \mathrm{~mm}$ ). Voxel-wise differences in BOLD contrast within the smoothed normalized images engendered by the different task conditions and trial types were examined using SPM. Individual design matrices, forming the basis for the multiple regression analysis of the functional imaging dataset, were constructed for each subject. For each of the numerical and physical tasks (modeled as separate sessions within the design), onset times of each trial were grouped according to trial type (congruent, neutral and incongruent). At these onsets, regressors (parametric modulators) were created for the numerical and physical distances (disparity) between components of each stimulus. Thus, regressors for both numerical and physical distances were included for congruent and incongruent trials. Error trials were entered into the analytic design as a separate regressor. Each trial were modeled as discrete events (delta function) with the temporal delay in BOLD response accommodated by a canonical hemodynamic response function with temporal and spatial derivatives as basis functions. Lastly, to independently test for brain areas where activity at the time of stimulus presentation reflected the magnitude of pupil response, a regressor was entered for each session in which each stimulus event was modeled as a delta function whose height was determined by the magnitude of evoked pupil change on that trial. This parametric event-related regressor was also convolved with a canonical function of hemodynamic lag. For individual subject data, regression analyses were performed using the General Linear Model to test for regressor-specific effects: $t$ tests of these regressors embodied implicit orthogonalization, enabling us to test variance explained uniquely by each regressor (partial correlation). Conjunction analyses were also used to derive measures of shared variance, notably, the commonality between error and pupil-related activity.

The significance of observations was determined across the group of 15 subjects, using standard methods, in second-level (random effects) analyses of the contrast images of individual subject effects. To ensure independence of our findings from the numerical and physical aspects of the tasks, we used conjunction analyses to test for conflict, error and pupil-related effects present in both tasks sessions (Price and Friston, 1997). Conjunction analysis of error and pupil-related responses at the second level was computed using identical methodology, i.e. thresholding SPM$\mathrm{t}$ images to a minimum $t$ value for conjoint significance. In these group analyses, threshold significance was set at $P<0.05$, corrected for whole brain for regions outside prefrontal cortex and ACC. Lateral prefrontal cortex (Brodmann's area 9 and inferior frontal gyrus), pre-SMA and rostral and dorsal ACC represented a priori regions of interest. Significance for these regions in second-level group analyses was thresholded initially at $P<0.001$, uncorrected, and survived correction for small volume ( 8 $\mathrm{mm}$ radius spheres centered on prefrontal and ACC coordinates published in previous studies [Carter et al., 1995, 1998; MacDonald et al., 2000; Bush et al., 2000; Braver et al., 2001; Botvinick et al., 2004; Menon et al., 2001; Milham et al., 2003]). In our anatomical descriptions, we use the term rostral ACC to refer to ACC regions anterior to the rostral extent of lateral ventricles and dorsal ACC to refer to ACC above the corpus callosum, posterior to rostral ACC (Botvinick et al., 2004).

\section{Results}

We scanned the subjects as they performed two numerical versions of the Stroop task, while simultaneously monitoring autonomic arousal responses from changes in pupil size. The 
Stroop tasks were extensively behaviorally piloted and required subjects to choose between a pair of numbers on each trial (Fig. 1). In one session (the Numerical task), the subject was instructed to select the numerically higher number while ignoring differences in the physical size of the stimuli. In the other session (the Physical task), the subject responded to the physically larger number while ignoring differences in numerical magnitudes. Conflict arose on trials where the selected number was smaller than the alternative in the distracting dimension (see Fig. 1). We measured congruency effects in reaction time separately for numeric and physical tasks. However, the focus was to determine autonomic and neural effects that were independent of task type. We therefore used conjunction analyses to determine effects that were present in both physical and numerical tasks, the rest of which are reported in the tables and figures.

The experimental design enabled us to obtain event-related fMRI data for conflict, non-conflict, neutral and error trials (where the subject made a wrong response). We also derived trial-by-trial measures of autonomic arousal generated from the recovery of the pupil light reflex, indexing pupil dilatation in response to 'arousing' trials (Fig. 2). We tested for brain regions where activity predicted the degree of evoked autonomic arousal by examining the correlation between activity at the onset of each trial and magnitude of the subsequent autonomic response. Multiple regression analyses were performed for individual brain imaging data and subsequent analyses of variance performed to test the significance of effects across the group.

\section{Behavioral and psychophysiological data}

Subjects made an average of 11.4 (SD \pm 7 ) errors per task session. Significantly more errors occurred on incongruent (conflict) trials $(F=56.10, P<0.001)$. There was no significant difference between Physical and Numerical tasks in number of errors $(F=3.34$, n.s.). Subjects' reaction times showed a response cost on incongruent trials and facilitation for congruent trials $(F=$ 112.7, $P<0.001 ; F=30.4, P<0.001$ ). For congruent versus incongruent trials, the mean reaction times for the Physical task were $553 \mathrm{~ms}$ versus $593 \mathrm{~ms}$ and for the Numerical task, $585 \mathrm{~ms}$ versus $652 \mathrm{~ms}$. The reaction time cost showed a reverse distance effect; that is, where competing disparities were closer and harder to resolve, reaction times were longer. Pupil responses indicated enhanced autonomic arousal to congruent, incongruent and error trials compared to neutral trials. Consistent with other observations (Hajcak et al., 2003), error trials were associated with greatest variability in evoked autonomic response. Although the largest evoked pupil responses occurred on error trials, it was our subjective impression that not all errors produced a distinct arousal reaction. Group ANOVA analyses of pupil arousal responses to the different trial types (congruent, incongruent, neutral and error) showed a trend level of significance $(F=2.9, P=0.07$, controlling for number of errors) (Fig. 2B).

\section{Functional imaging data}

Functional imaging data analysis at the first level enabled us to model neural responses associated with congruent, incongruent and error trials independent of stimulus-induced arousal responses. Second level analyses were conducted to test for brain regions independently associated with conflict, error and autonomic (pupil) response. We modeled these effects separately for the
Physical and Numerical Stroop tasks then applied conjunction analyses to ensure that we identified activity that was common to both tasks.

The contrast between incongruent versus congruent stimuli represents the conflict between response options that is associated with a reaction time (RT) cost. Enhanced activity associated with the main effect of conflict was expressed in lateral and medial prefrontal cortices, notably, the right lateral prefrontal cortex (inferior frontal gyrus; peak $x, y, z$ coordinates: 58,14,6 and $54,12,24)$, pre-supplementary motor area (pre-SMA, 12,18,48), premotor cortex $(22,2,54)$ and eight voxels in dACC $(6,22,38)$ (Table 1; Figs. 3A,B).

Error trials, compared to all correct trials, were associated with enhanced activity in bilateral insula cortex, right parietal cortex (extending to middle temporal gyrus), right lateral prefrontal cortex (inferior frontal gyrus; 38,28,2), medial thalamus and dorsal pons (Fig. 3B). Notably, activity was also enhanced in $\mathrm{rACC}$ adjacent to more rostral activity attributable to evoked autonomic response $(x, y, z$ coordinates $6,28,18)$ (see below).

Across the two Stroop tasks, trial-by-trial variation in evoked autonomic pupil response correlated with bilateral activity in $\mathrm{rACC}$ (peak coordinates $0,44,14$ and 2,34,14) (Fig. 3C). As noted, this autonomic-related activity was adjacent but anterior to rACC activity attributable to error responses and distinct in location from pre-SMA activity attributable to correct conflict processing (Table 1, Fig. 3).

In analyses of individual subject data, specified contrasts (that subsequently contribute to the group analysis) test for significant activity, additional to that explained by the rest of experimental effects. Thus, fMRI analyses of arousal-related and error-related activity do not reflect their shared variance due to this implicit orthogonalization. We therefore tested, using a conjunction analysis, for brain regions where activity was enhanced during error trials and where activity predicted the magnitude of evoked autonomic arousal response. Activity attributable to both error and arousal was expressed in $\operatorname{rACC}(0,25,20)$ and $\mathrm{dACC}(6,21,39)$ regions, extending caudally and superiorly to mediodorsal prefrontal cortex $(2,21,52)$. Notably, this did not include the pre-SMA activity attributable to correct conflict processing. Significant shared activity was also observed in the brainstem, at the level of the dorsal pons (Table 1, Fig. 4).

\section{Discussion}

Our findings implicate adjacent contiguous rostral and dorsal regions of ACC in the control of autonomic arousal states (here indexed by using pupillometry) and in error processing. These findings suggest that these ACC regions support an integration of error processing with generation of states of autonomic arousal. We note that the variance in the magnitude of evoked autonomic changes in pupil response was maximal in error trials.

The selective association between autonomic arousal responses (vagal bradycardia and electrodermal sympathetic arousal) and error trials in a Stroop task has been reported in a combined psychophysiological and electrophysiological experiment (Hajcak et al., 2003). Additionally, some versions of the Stroop task may elicit sympathetic pupil dilatation even during conflict trials (Siegle et al., 2004; Brown et al., 1999). Within our experiment, we observed a trend for a greater overall autonomic arousal response and increased variability in autonomic arousal during error trials, 
Table 1

Activity related to Stroop task performance

\begin{tabular}{|c|c|c|c|}
\hline Location & Side & Coordinates & $Z$ score \\
\hline \multicolumn{4}{|c|}{ Activity related to conflict (incongruent > congruent correct trials) independent of autonomic response } \\
\hline Supplementary motor area & $\mathrm{R}$ & $12,18,48$ & 5.14 \\
\hline Premotor cortex & $\mathrm{R}$ & $28,2,54$ & 4.14 \\
\hline \multirow[t]{2}{*}{ Right inferior frontal gyrus } & $\mathrm{R}$ & $58,14,6$ & 3.81 \\
\hline & $\mathrm{R}$ & $54,12,24$ & 3.72 \\
\hline Dorsal anterior cingulate cortex & - & $6,22,38$ & 3.46 \\
\hline \multicolumn{4}{|c|}{ Activity related to error processing (error > correct trials) independent of autonomic response } \\
\hline Temporoparietal junction & $\mathrm{R}$ & $60,-44,12$ & 5.98 \\
\hline Superior temporal gyrus & $\mathrm{R}$ & $48,-30,-8$ & 5.97 \\
\hline Cerebellar cortex & $\mathrm{L}$ & $-18,-74,-32$ & 5.55 \\
\hline Inferior frontal gyrus & $\mathrm{R}$ & $34,28,2$ & 4.85 \\
\hline \multirow[t]{2}{*}{ Anterior insula } & $\mathrm{R}$ & $34,28,2$ & 4.85 \\
\hline & $\mathrm{L}$ & $-32,22,-2$ & 4.64 \\
\hline Dorsal pons & - & $2,-28,-36$ & 4.82 \\
\hline Inferior frontal gyrus & $\mathrm{R}$ & $52,24,6$ & 4.17 \\
\hline Rostral anterior cingulate cortex & - & $6,28,18$ & 4.10 \\
\hline \multicolumn{4}{|c|}{ Activity attributable to trial-by-trial variation in sympathetic pupil response independent of error and conflict } \\
\hline \multirow[t]{2}{*}{ Rostral anterior cingulate cortex } & - & $0,44,14$ & 4.73 \\
\hline & - & $2,34,14$ & 4.29 \\
\hline Motor cingulate cortex & & $-2,-10,42$ & 4.01 \\
\hline \multicolumn{4}{|c|}{ Activity reflecting both error processing and sympathetic arousal (conjunction analysis of main effects) } \\
\hline Rostral anterior cingulate cortex & - & $0,25,20$ & 6.25 \\
\hline Dorsal anterior cingulate cortex & - & $6,21,39$ & 5.54 \\
\hline Medial prefrontal cortex (superior frontal gyrus) & - & $2,21,52$ & 7.60 \\
\hline Brainstem (dorsal pons) & - & $4,-28,-30$ & 4.51 \\
\hline \multirow[t]{2}{*}{ Frontal insula } & $\mathrm{R}$ & $34,16,-11$ & 6.30 \\
\hline & $\mathrm{L}$ & $34,18,-11$ & 6.10 \\
\hline
\end{tabular}

driven by robust responses in some (but not all) error trials. This pattern of response was not observed for conflict trials. It has been suggested that awareness of errors in performance underlies generation of strong error-related autonomic responses and influences associated magnitude of error-related ERPs that putatively originate in ACC (Hajcak et al., 2003). We were unable to segregate error trials on the basis of subjective awareness, however, other studies report ACC sensitivity to subjective awareness of internal or external processes (Dehaene et al., 2003; Critchley et al., 2004), an important factor when considering the role of ACC regions in expression of subjective pain and anxiety (Rainville, 2002; Ploghaus et al., 1999, 2003). It remains uncertain whether the subjects' awareness of the error drives autonomic arousal in the pupil response. Certainly, the late recovery of the light reflex, taken in this study as our autonomic measure, may well be influenced by subjective awareness (the ERP correlating with awareness and arousal occurs with $200 \mathrm{~ms}$ of the error (Hajcak et al., 2003). However, error-related blunting of the light reflex (attenuation of the parasympathetic light response) influenced the magnitude of our arousal measure and was at times observed to occur within $200 \mathrm{~ms}$ of the stimulus presentation. It is therefore possible that error commission may share in part a common neural substrate, with perhaps magnitude of autonomic responses reinforcing error awareness to influence response on subsequent trials. It is noteworthy that same regions of dorsal ACC regions have been implicated in both error detection and error correction (Fiehler et al., 2004). Unfortunately, we were unable to demonstrate a consistent relationship between autonomic response magnitude and reaction time on the following trial, even for error trials.

A key hypothesis underlying the present study was that activity in ACC, the putative generator of peripheral arousal responses, would relate most robustly to event-related sympathetic pupil measures rather than to error or conflict processing alone. In fact, we initially observed dissociation between $\mathrm{rACC}$ activity predictive of sympathetic influences on pupil response and adjacent (still rostral) ACC activity reflecting error processing. One interpretation is that autonomic arousal is independently controlled from error processing within the ACC region. Moreover, this functional segregation of a rostral 'autonomic' from a more posterior 'cognitive' region of ACC is broadly consistent with earlier arguments for segregation of emotional and cognitive sectors of ACC (e.g. Bush et al., 2000) and might appear to run counter to our earlier 'unitary' account that ACC translates cognitive processing into bodily arousal responses (Critchley et al., 2003). Nevertheless, as in earlier studies (Hajcak et al., 2003), error trials tended to predict autonomic arousal responses, therefore it is likely that the segregation of one from the other reflects implicit orthogonalization within the multiple regression analyses (that is, arousal is treated as a confound in identifying activity related to error processing and vice versa). Using a conjunction analysis approach to overcome this limitation, we demonstrate activity in a band of ACC (extending from rostral ACC, through AACC to caudal ACC) attributable conjointly to both error processing and evoked autonomic arousal. This observation is consistent with the proposal that efferent autonomic responses evoked by cognitive processes have their 
A.
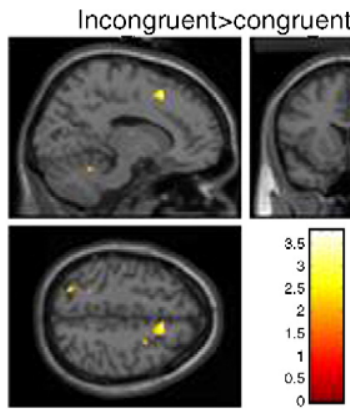

B.
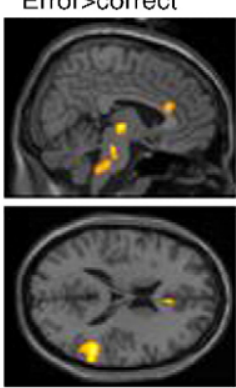
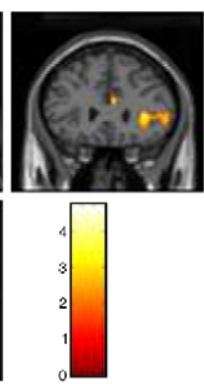

Incongruent>congruent
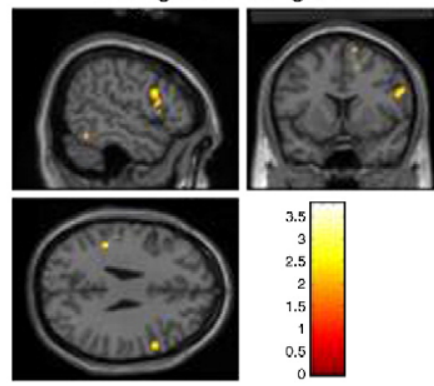

Error $>$ correct
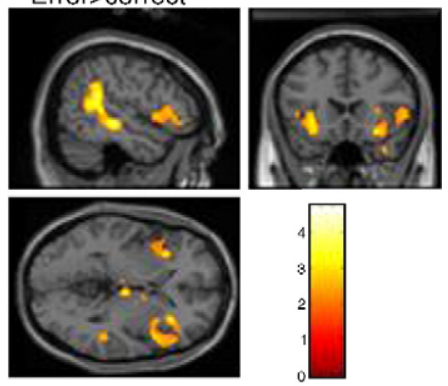

C.

Activity related to sympathetic influences on pupil response
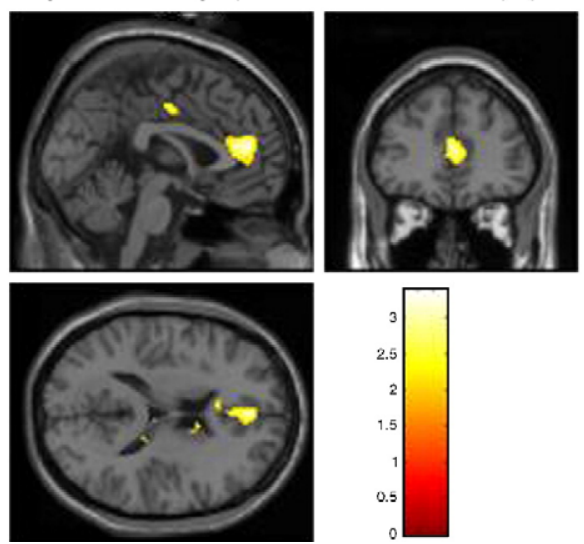

Fig. 3. Brain activity associated with conflict and error processing in task performance and predicting sympathetic arousal manifest in pupil responses. Individual group analyses modeled trial types, including errors, for both Numerical and Physical task sessions. These first level analyses also modeled eventrelated activity predicting sympathetic (pupil) arousal as a continuous regressor for each task session. The figure shows data from separate second-level group analyses of the effects of conflict, error processing and pupillary arousal response. Activity common to Numerical and Physical tasks (determined using conjunction analyses) is plotted on a normalized template brain at a threshold of $P<0.001$, uncorrected. (A) Plots of activity related to conflict processing, i.e. the difference in activity between incongruent compared to congruent correct trials. The plots highlight on two sets of orthogonal sections the enhanced activity observed in dACC/pre-SMA and right inferior convexity (inferior frontal gyrus) during conflict trials in both Numerical and Physical Stroop tasks. (B) Brain activity associated with error responses (compared to correct trials). The plots highlight on two sets of orthogonal sections the enhanced activity observed in right lateral parietal/STS cortex and right inferior convexity (inferior frontal gyrus), perhaps reflecting response monitoring and error-induced augmentation of visual attention. Despite controlling for pupil-related arousal, error trials were also associated with enhanced activity in regions implicated in somatic arousal including brainstem (dorsal pons), medial thalamus, bilateral anterior insula and a discrete region of rostral ACC. (C) Brain activity independently predicting trial-induced variations in pupil diameter, reflecting sympathetic influence on pupil response. The plots show on orthogonal sections enhanced activity correlating with this measure of autonomic arousal in rostral ACC anterior to error-related activity.

origin in ACC activity (Hajcak et al., 2003). Nevertheless, one ACC region remained uniquely sensitive to errors, while rostral ACC reflected non-error-related autonomic influences on pupil response.

The role of ACC activity in generating specific patterns of autonomic response is unclear since arousal may not represent a unitary concept. There is a degree of organ specificity in peripheral autonomic arousal responses, both within and between sympathetic and parasympathetic axes. Orienting arousal responses may be manifest as a parasympathetic cardiac bradycardia and enhanced sympathetic electrodermal activity (Porges, 1995; Mathias and Bannister, 1999; Morrison, 2001). Pupil light reflex, a typically parasympathetic process, is modulated by sympathetic 'emotional' arousal (Bitsios et al., 1998). In general, different psychophysiological indices are correlated, though different response patterns can occur across autonomic axes (Cacioppo et al., 1994; Ekman et al., 1983). In neuroimaging studies, enhanced electrodermal sympathetic activity during motivational or anticipatory processing is associated with increased activity in rostral ACC, close to the 


\section{Medial cerebral activity associated with autononmic arousal, error and conflict during Stroop task performance}

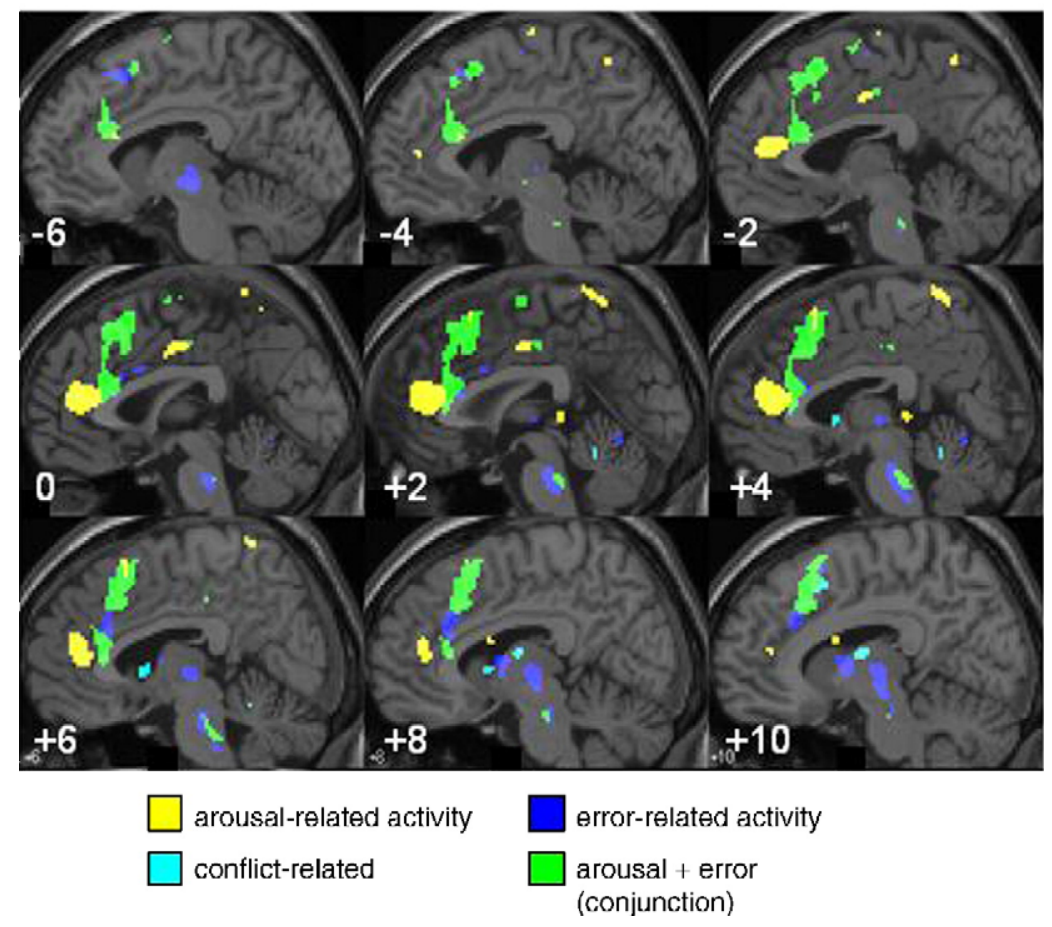

Fig. 4. Locations of peak activity in medial frontal and ACC cortices (and other medial brain regions) related independently to autonomic response (yellow), error processing (blue) and conflict processing (cyan). In addition, the location of common activity shared between error processing and autonomic response (i.e. predictive of sympathetic influence on pupil) is plotted in green, tested for using a conjunction analysis of main effects. Effects are plotted on sagittal sections of a normalized structural MRI derived from one subject at a threshold significance of $P<0.001$, uncorrected. Much of the medial prefrontal/dACC error-related activity also predicts autonomic pupil effects and lies immediately caudal to a rostral ACC region predicting pupil effects independently of error and conflict. Conflict processing (in correct trials) also enhanced dACC and pre-SMA activity contiguous with error/arousal-related activity. The lateral ( $y$ ) coordinates of the sections are given in millimeters from the midline in standard space.

genu of the corpus callosum (Critchley et al., 2001b). Cardiovascular arousal in response to physical and cognitive challenge is associated with enhanced activity more caudally in dACC (Critchley et al., 2000, 2001a, 2003). Our observations in the present study suggest that enhanced activity attributable to a pupillary index of sympathetic arousal which lies close to the same rACC region implicated emotional or motivational arousal. However, when examined in conjunction with error-related processing, the pattern of activity, including $\mathrm{dACC}$, is similar to that previously related to cardiovascular arousal (Critchley et al., 2000, 2001a, 2003). While it is probable that the psychological context determines the cingulate sector driving a bodily response, it is plausible that these differences also reflect differences in the pattern of arousal across autonomic axes engendered by the task.

Error responses were associated with enhanced neural activity in other brain regions, notably dorsal pons and bilateral insula. Within these regions, there is activity that also reflects sympathetic pupil response in the conjunction analysis with error. However, the error-related activity extends beyond the shared activity in these regions. Enhanced activity in dACC, insula and dorsal pons is associated with induced states of cardiovascular arousal (Critchley et al., 2000, 2003; Williamson et al., 2001). The activity in insula cortex may specifically reflect interoceptive processes, including the central representation of peripheral autonomic arousal (Craig, 2003; Critchley et al., 2003, 2004). It was unfortunate that we did not simultaneously record cardiovascular and pupil responses in the present study so we cannot comment if cardiovascular arousal responses might account for a further proportion of error-related activity. However, it is noteworthy that the robust association between error trials and cardiac arousal responses in the study of Hajcak et al. (2003) would suggest such a possibility.

In functional imaging studies, the medial frontal region most commonly associated with conflict and error detection is pre-SMA, located caudally above the cingulate sulcus next to the SMA (Ullsperger and von Cramon, 2001; Rushworth et al., 2003). Nevertheless, the role of dACC in these 'cognitive' processes is much emphasized (Carter et al., 1995, 1998; MacDonald et al., 2000; Bush et al., 2000; Braver et al., 2001; Botvinick et al., 2001, 2004; Kerns et al., 2004). We also observed some enhanced activity across both pre-SMA and AACC in association with conflict. Activity anterior to conflict-related area was modulated by both error and autonomic arousal, suggesting that activity within adjacent medial frontal regions may be sensitive to both conflict and error/arousal (e.g. Kerns et al., 2004). Conflict-related dACC activity is reported to influence the activity of dorsolateral prefrontal cortex expressed on subsequent trials, predicting implicit adjustments in reaction time (Kerns et al., 2004). One interpretation is that pre-SMA/dACC activation engages 'cognitive control' functions (presumably supported by dorsolateral prefrontal cortices) to resolve competition between responses or processed information. In part, this may be achieved by an efference copy/ 
comparator mechanism implemented by dACC-prefrontal interactions (Cohen and Huston, 1994; Botvinick et al., 2001, 2004; Siegle et al., 2004). However, a number of related processes are also likely to contribute to adaptive modulation of ongoing behavior, including dopaminergic error signaling (Cohen et al., 2002; Schultz, 2002) and re-representation of stimulus-evoked autonomic responses within medial and lateral prefrontal cortex (Damasio, 1996).

Profound executive disturbances may follow dorsal and lateral prefrontal damage, whereas deficits following dACC damage are relatively modest (Turken and Swick, 1999; Stuss et al., 2001; Rushworth et al., 2004). It would appear that in some instances performance on cognitive control or executive function tasks can be entirely normal in the presence of large bilateral lesions of ACC (Critchley et al., 2003; Fellows and Farah, 2005) These observations support a proposal that lateral prefrontal cortex, rather than ACC, computes and represents information about conflict and error to permit behavioral adjustments (Gehring and Knight, 2000). The timing of ERP responses suggests that $\mathrm{dACC}$ is one of the first regions to process conflict or error (Dehaene et al., 1994), however, lesion data suggest that cognitive control mechanisms are not critically dependent on dACC. Rather than reflecting an executive function, ACC responses may in fact represent a low-level orienting signal to atypical trials.

Our data add to evidence that lateral prefrontal cortex is critically involved in processing conflict and error. We observed enhanced inferior frontal gyrus activity in both the conflict and error trials. Lateral prefrontal cortex is commonly activated in task situations involving monitoring and response selection (Fletcher and Henson, 2001), but many studies implicate middle, rather than inferior, frontal gyrus in conflict monitoring and response adjustment (Kerns et al., 2004; Paulus et al., 2002). Nevertheless, coactivation of inferior frontal gyrus and $\mathrm{rACC}$ is reported during performance of Stroop tasks where there is competition from taskirrelevant distractor stimuli including threat (Milham et al., 2003; Bishop et al., 2004) and also during error trials in go/no-go tasks (Menon et al., 2001). The observation that damage to lateral prefrontal cortical regions can impair the specificity of midline ERPs to error trials, while incidentally not impairing attentional switching, adds further evidence for the importance of lateral prefrontal cortex, and perhaps an incidental role of ACC, in cognitive control (Gehring and Knight, 2000, 2002).

In summary, we observed rACC activity relating to variability in an evoked pupil measure of autonomic arousal during Stroop task performance, independently of conflict and error processing. Activity uniquely attributable to error-processing was observed in an adjacent ACC region and, when considered in conjunction with autonomic arousal, activity through much of rACC and AACC was shown to be sensitive to error and to predict the evoked autonomic responses. These data converge with other lesion and neuroimaging evidence using multiple task modalities and autonomic indices to endorse a psychophysiological account of ACC function wherein ACC provides an interface between high-level appraisal and low-level visceral systems that may influence behavioral adaptation.

\section{Acknowledgment}

This research was supported by the Wellcome Trust via a program grant to RJD and a fellowship to HDC.

\section{References}

An, X., Bandler, R., Ongur, D., Price, J.L., 1998. Prefrontal cortical projections to longitudinal columns in the midbrain periaqueductal gray in macaque monkeys. J. Comp. Neurol. 401, 455-479.

Barbas, H., Saha, S., Rempel-Clower, N., Ghashghaei, T., 2003. Serial pathways from primate prefrontal cortex to autonomic areas may influence emotional expression. BMC Neurosci. 4, 25.

Bishop, S., Duncan, J., Brett, M., Lawrence, A.D., 2004. Prefrontal cortical function and anxiety, controlling attention to threat-related stimuli. Nat. Neurosci. 7, 184-188.

Bitsios, P., Szabadi, E., Bradshaw, C.M., 1998. Sensitivity of the fear-inhibited light reflex to diazepam. Psychopharmacology 135, $93-98$.

Botvinick, M.M., Braver, T.S., Barch, D.M., Carter, C.S., Cohen, J.D., 2001. Conflict monitoring and cognitive control. Psychol. Rev. 108, 624-652.

Botvinick, M.M., Cohen, J.D., Carter, C.S., 2004. Conflict monitoring and anterior cingulate cortex: an update. Trends Cogn. Sci. 8, $539-546$.

Braver, T.S., Barch, D.M., Gray, J.R., Molfese, D.L., Snyder, A., 2001. Anterior cingulate cortex and response conflict, effects of frequency, inhibition and errors. Cereb. Cortexfs 11, 825-836.

Brodmann, K., 1909. Vergleichende lokalisationslehre der Grosshirnrinde in ihren Prinzipien dargestellt auf Grund des Zellenbaues. J.A. Barth, Leipzig.

Brown, G., Kinderman, S., Siegle, G.J., Granholm, E., Wong, E.C., Buxton, R.B., 1999. Brain activation and pupil response during covert performance of the Stroop color word task. J. Int. Neuropsychol. Soc. $54,308-319$

Büchel, C., Morris, J., Dolan, R.J., Friston, K.J., 1998. Brain systems mediating aversive conditioning, an event-related fMRI study. Neuron 20, 947-957.

Bush, G., Luu, P., Posner, M.I., 2000. Cognitive and emotional influences in anterior cingulate cortex. Trends Cogn. Sci. 4, 215-222.

Cacioppo, J.T., Uchino, B.N., Berntson, G.G., 1994. Individual differences in the autonomic origins of heart rate reactivity, the psychometrics of respiratory sinus arrhythmia and preejection period. Psychophysiology $31,412-419$.

Carter, C.S., Mintun, M., Cohen, J.D., 1995. Interference and facilitation effects during selective attention, an H215O PET study of Stroop task performance. NeuroImage 2, 264-272.

Carter, C.S., Braver, T.S., Barch, D.M., Botvinick, M.M., Noll, D., Cohen, J.D., 1998. Anterior cingulate cortex, error detection, and the online monitoring of performance. Science 280, 747-749.

Cohen, J.D., Huston, T.A., 1994. Progress in the use of interactive models for understanding attention and performance. In: Umilta, C., Moscovitch, M. (Eds.), Attention and Performance, vol. XV. MIT, Cambridge, MA, pp. 453-456.

Cohen, J.D., Braver, T.S., Brown, J.W., 2002. Computational perspectives on dopamine function in prefrontal cortex. Curr. Opin. Neurobiol. 12, $223-229$

Craig, A.D., 2003. Interoception, the sense of the physiological condition of the body. Curr. Opin. Neurobiol. 13, 500-505.

Critchley, H.D., Corfield, D.R., Chandler, M.P., Mathias, C.J., Dolan, R.J., 2000. Cerebral correlates of autonomic cardiovascular arousal. A functional neuroimaging investigation. J. Physiol. (London) 523, $259-270$.

Critchley, H.D., Mathias, C.J., Dolan, R.J., 2001a. Neural correlates of first and second-order representation of bodily states. Nat. Neurosci. 2, 207-212.

Critchley, H.D., Mathias, C.J., Dolan, R.J., 2001b. Neural activity in the human brain relating to uncertainty and arousal during anticipation. Neuron 29, 537-545.

Critchley, H.D., Mathias, C.J., Dolan, R.J., 2002. Fear-conditioning in humans. The influence of awareness and arousal on functional neuroanatomy. Neuron 33, 653-663. 
Critchley, H.D., Mathias, C.J., Josephs, O., O'Doherty, J., Zanini, S., Dewar, B.K., Cipolotti, L., Shallice, T., Dolan, R.J., 2003. Human cingulate cortex and autonomic control, converging neuroimaging and clinical evidence. Brain 126, 2139-2152.

Critchley, H.D., Wiens, S., Rotshtein, P., Ohman, A., Dolan, R.J., 2004. Neural systems supporting interoceptive awareness. Nat. Neurosci. 7, $189-195$.

Damasio, A.R., 1996. The somatic marker hypothesis and the possible functions of the prefrontal cortex. Philos. Trans. R. Soc. Lond., Ser. B Biol. Sci. 351, 1413-1420.

Dehaene, S., Posner, M.I., Tucker, D.M., 1994. Localization of a neural system for error detection and compensation. Psychol. Sci. 5, $303-305$

Dehaene, S., Artiges, E., Naccache, L., Martelli, C., Viard, A., Schurhoff, F., Recasens, C., Martinot, M.L., Leboyer, M., Martinot, J.L., 2003. Conscious and subliminal conflicts in normal subjects and patients with schizophrenia, the role of the anterior cingulate. Proc. Natl. Acad. Sci. U. S. A. $100,13722-13727$

Deichmann, R., Gottfried, J.A., Hutton, C., Turner, R., 2003. Optimized EPI for fMRI studies of the orbitofrontal cortex. NeuroImage 19, 430-441.

Dum, R.P., Strick, P.L., 1993. Cingulate motor areas. In: Vogt, B.A., Graybiel, M. (Eds.), Cingulate Cortex and Limbic Thalamus: A Comprehensive Handbook. Birkhauser, Boston, pp. 415-441.

Duncan, J., Owen, A.M., 2000. Common regions of the human frontal lobe recruited by diverse cognitive demands. Trends Neurosci. 23, 475-483.

Ekman, P., Levenson, R.W., Friesen, W.V., 1983. Autonomic nervous system activity distinguishes among emotions. Science $221,1208-1210$.

Fellows, L.K., Farah, M.J., 2005. Is anterior cingulate cortex necessary for cognitive control? Brain 128, 788-796.

Fiehler, K., Ullsperger, M., von Cramon, D.Y., 2004. Neural correlates of error detection and error correction, is there a common neuroanatomical substrate? Eur. J. Neurosci. 9, 3081.

Fletcher, P.C., Henson, R.N., 2001. Frontal lobes and human memory: insights from functional neuroimaging. Brain 124, 849-881.

Fredrikson, M., Furmark, T., Olsson, M.T., Fischer, H., Andersson, J., Langstrom, B., 1998. Functional neuroanatomical correlates of electrodermal activity: a positron emission tomographic study. Psychophysiology $35,179-185$.

Frith, C., 2001. A framework for studying the neural basis of attention. Neuropsychologia 39, 1367-1371.

Gehring, W.J., Knight, R.T., 2000. Prefrontal-cingulate interactions in action monitoring. Nat. Neurosci. 3, 516-520.

Gehring, W.J., Knight, R.T., 2002. Lateral prefrontal damage affects processing selection but not attention switching. Brain Res. Cogn. Brain Res. 13, 267-279.

Gianaros, P.J., Van Der Veen, F.M., Jennings, J.R., 2004. Regional cerebral blood flow correlates with heart period and high-frequency heart period variability during working-memory tasks: implications for the cortical and subcortical regulation of cardiac autonomic activity. Psychophysio$\operatorname{logy} 41,521-530$.

Gianaros, P.J., May, J.C., Siegle, G.J., Jennings, J.R., 2005. Is there a functional neural correlate of individual differences in cardiovascular reactivity? Psychosom. Med. 67, 31-39.

Gusnard, D.A., Akbudak, E., Shulman, G.L., Raichle, M.E., 2001. Medial prefrontal cortex and self-referential mental activity, relation to a default mode of brain function. Proc. Natl. Acad. Sci. U. S. A. $27,4259-4264$.

Hajcak, G., McDonald, N., Simons, R.F., 2003. To err is autonomic, errorrelated brain potentials. ANS activity, and post-error compensatory behavior. Psychophysiology 40, 895-903.

Holroyd, C.B., Nieuwenhuis, S., Yeung, N., Nystrom, L., Mars, R.B., Coles, M.G., Cohen, J.D., 2004. Dorsal anterior cingulate cortex shows fMRI response to internal and external error signals. Nat. Neurosci. 7, 497-498.

Kerns, J.G., Cohen, J.D., MacDonald III, A.W., Cho, R.Y., Stenger, V.A., Carter, C.S., 2004. Anterior cingulate conflict monitoring and adjustments in control. Science 303, 1023-1026.
Kiehl, K.A., Liddle, P.F., Hopfinger, J.B., 2000. Error processing and the rostral anterior cingulate, an event-related fMRI study. Psychophysiology $37,216-223$.

Luppino, G., Rozzi, S., Calzavara, R., Matelli, M., 2003. Prefrontal and a granular cingulate projections to the dorsal premotor areas F2 and F7 in the macaque monkey. Eur. J. Neurosci. 17, 559-578.

MacDonald, A.W., Cohen, J.D., Stenger, V.A., Carter, C.S., 2000. Dissociating the role of the dorsolateral prefrontal and anterior cingulate cortex in cognitive control. Science 288, 1835-1838.

Mathias, C.J., Bannister, R., 1999. Autonomic failure. A Textbook of Clinical Disorders of the Autonomic Nervous System, 4th ed. Oxford Univ. Press, Oxford, pp. 169-195.

Matthews, S.C., Paulus, M.P., Simmons, A.N., Nelesen, R.A., Dimsdale, J.E., 2004. Functional subdivisions within anterior cingulate cortex and their relationship to autonomic nervous system function. NeuroImage 22, $1151-1156$.

Menon, V., Adleman, N.E., White, C.D., Glover, G.H., Reiss, A.L., 2001. Error-related brain activation during a Go/NoGo response inhibition task. Hum. Brain Mapp. 12, 131-143.

Milham, M.P., Banich, M.T., Barad, V., 2003. Competition for priority in processing increases prefrontal cortex's involvement in top-down control: an event-related fMRI study of the Stroop task. Brain Res. Cogn. Brain Res. 17, 212-222.

Morecraft, R.J., Van Hoesen, G.W., 1998. Convergence of limbic input to the cingulate motor cortex in the rhesus monkey. Brain Res. Bull. 45, $209-232$.

Morris, R., Pandya, D.N., Petrides, M., 1999. Fiber system linking the middorsolateral frontal cortex with the retrosplenial/presubicular region in the rhesus monkey. J. Comp. Neurol. 407, 183-192.

Morrison, S.F., 2001. Differential control of sympathetic outflow. Am. J. Physiol.: Regul., Integr. Comp. Physiol. 281, R683-R698.

Nagai, Y., Critchley, H.D., Featherstone, E., Trimble, M.R., Dolan, R.J., 2004a. Activity in ventromedial prefrontal cortex covaries with sympathetic skin conductance level SCL, a physiological account of a "default mode" of brain function. NeuroImage 22, 243-251.

Nagai, Y., Critchley, H.D., Featherstone, E., Fenwick, P., Trimble, M.R., Dolan, R.J., 2004b. Brain activity relating to the contingent negative variation: an fMRI investigation. NeuroImage 21, 1232-1241.

Öngür, D., Price, J.L., 2000. The organization of networks within the orbital and medial prefrontal cortex of rats, monkeys and humans. Cereb. Cortex. 10, 206-219.

Öngür, D., Ferry, A.T., Price, J.L., 2003. Architectonic subdivision of the human orbital and medial prefrontal cortex. J. Comp. Neurol. 460, $425-449$.

Paulus, M.P., Hozack, N., Frank, L., Brown, G.G., 2002. Error rate and outcome predictability affect neural activation in prefrontal cortex and anterior cingulate during decision-making. NeuroImage 15 , $836-846$.

Paus, T., Koski, L., Caramanos, Z., Westbury, C., 1998. Regional differences in the effects of task difficulty and motor output on blood flow response in the human anterior cingulate cortex, a review of 107 PET activation studies. NeuroReport 9, R37-R47.

Paus, T., Castro-Alamancos, M.A., Petrides, M., 2001. Cortico-cortical connectivity of the human mid-dorsolateral frontal cortex and its modulation by repetitive transcranial magnetic stimulation. Eur. J. Neurosci. 14, 1405-1411.

Ploghaus, A., Tracey, I., Gati, J.S., Clare, S., Menon, R.S., Matthews, P.M., Rawlins, J.N., 1999. Dissociating pain from its anticipation in the human brain. Science 284, 1979-1981.

Ploghaus, A., Becerra, L., Borras, C., Borsook, D., 2003. Neural circuitry underlying pain modulation: expectation, hypnosis, placebo. Trends Cogn. Sci. 7, 197-200.

Porges, S.W., 1995. Orienting in a defensive world: mammalian modification of our evolutionary heritage. A polyvagal theory. Psychophysiology $32,301-318$.

Posner, M.I., Petersen, S.E., 1999. The attention system of the human brain. Annu. Rev. Neurosci. 13, 25-42. 
Price, C., Friston, K.J., 1997. Cognitive conjunction: a new approach to brain activation experiments. NeuroImage 5, 261-270.

Raichle, M.E., MacLeod, A.M., Snyder, A.X., Powers, W.J., Gusnard, D.A., Shulman, G.L., 2001. A default mode of brain function. Proc. Natl. Acad. Sci. U. S. A. 16, 676-682.

Rainville, P., 2002. Brain mechanisms of pain affect and pain modulation. Curr. Opin. Neurobiol. 12, 195-204.

Rushworth, M.F., Hadland, K.A., Gaffan, D., Passingham, R.E., 2003. The effect of cingulate cortex lesions on task switching and working memory. J. Cogn. Neurosci. 15, 338-353.

Rushworth, M.F., Walton, M.E., Kennerley, S.W., Bannerman, D.M., 2004. Action sets and decisions in the medial frontal cortex. Trends Cogn. Sci. $8,410-417$.

Schultz, W., 2002. Getting formal with dopamine and reward. Neuron 36, $241-263$.

Siegle, G.J., Steinhauer, S.R., Thase, M., 2004. Pupil assessment and computational modeling of the Stroop task in depression. Int. J. Psychophysiol. 52, 63-76.

Simpson, J.R., Drevets, W.C., Snyder, A.Z., Gusnard, D.A., Raichle, M.E., 2001. Emotion-induced changes in human medial prefrontal cortex: I During cognitive task performance. Proc. Natl. Acad. Sci. U. S. A. 16, 683-687.

Smith, S., 1992. Pupil function tests and disorders. In: Bannister, R., Mathias, C.J. (Eds.), Autonomic Failure, A Textbook of Clinical Disorders of the Autonomic System, 3rd ed. Oxford Univ. Press, Oxford, pp. 421-441.

Stemmer, B., Segalowitz, S.J., Witzke, W., Schonle, P.W., 2004. Error detection in patients with lesions to the medial prefrontal cortex: an ERP study. Neuropsychologia 42, 118-130.

Stuss, D.T., Floden, D., Alexander, M.P., Levine, B., Katz, D., 2001. Stroop performance in focal lesion patients, dissociation of processes and frontal lobe lesion location. Neuropsychologia 39, $771-786$.
Swick, D., Turken, A.U., 2002. Dissociation between conflict detection and error monitoring in the human anterior cingulate cortex. Proc. Natl. Acad. Sci. U. S. A. 99, 16354-16359.

Tachibana, Y., Nambu, A., Hatanaka, N., Miyachi, S., Takada, M., 2004. Input-output organization of the rostral part of the dorsal premotor cortex, with special reference to its corticostriatal projection. Neurosci. Res. 481, 45-57.

Takada, M., Nambu, A., Hatanaka, N., Tachibana, Y., Miyachi, S., Taira, M., Inase, M., 2004. Organization of prefrontal outflow toward frontal motorrelated areas in macaque monkeys. Eur. J. Neurosci. 19, 3328-3342.

Tang, J., Critchley, H., Glaser, D., Dolan, R., Butterworth, B., submitted for publication. Imaging informational conflict: an fMRI study of numerical Stroop.

Turken, A.U., Swick, D., 1999. Response selection in the human anterior cingulate cortex. Nat. Neurosci. 2, 920-924.

Ullsperger, M., von Cramon, D.Y., 2001. Subprocesses of performance monitoring: a dissociation of error processing and response competition revealed by event-related fMRI and ERPs. NeuroImage 14, 1387-1401.

Vilensky, J.A., van Hoesen, G.W., 1981. Corticopontine projections from the cingulate cortex in the rhesus monkey. Brain Res. 205, 391-395.

Vogt, B.A., Nimchinsky, E.A., Vogt, L.J., Hof, P.R., 1995. Human cingulate cortex, surface features, flat maps, and cytoarchitecture. J. Comp. Neurol. 359, 490-506.

Vogt, B.A., Berger, G.R., Derbyshire, S.W.G., 2003. Structural and functional dichotomy of human midcingulate cortex. Eur. J. Neurosci. $18,3134-3144$.

Williamson, J.W., McColl, R., Mathews, D., Mitchell, J.H., Raven, P.B., Morgan, W.P., 2001. Hypnotic manipulation of effort sense during dynamic exercise: cardiovascular responses and brain activation. J. Appl. Physiol. 90, 1392-1399.

Zahn, T.P., Grafman, J., Tranel, D., 1999. Frontal lobe lesions and electrodermal activity, effects of significance. Neuropsychologia 37, $1227-1241$. 